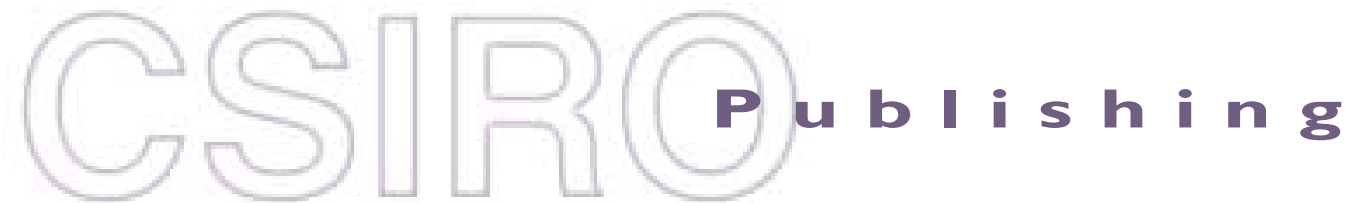

\section{Publications of the Astronomical Society of Australia}

Volume 19, 2002

(C) Astronomical Society of Australia 2002

An international journal of astronomy and astrophysics

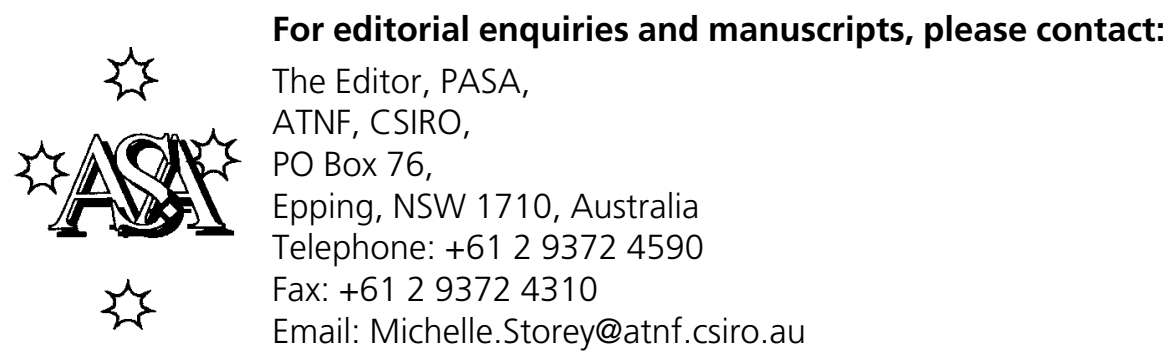

For general enquiries and subscriptions, please contact: CSIRO Publishing PO Box 1139 (150 Oxford St)

Collingwood, Vic. 3066, Australia

Telephone: +6139662 7666

Fax: +61 396627555

Email: publishing.pasa@csiro.au

C S I RO

PUBLISHING Published by CSIRO Publishing

for the Astronomical Society of Australia

www.publish.csiro.au/journals/pasa 


\title{
The Size of IDV Jet Cores
}

\author{
T. Beckert, T. P. Krichbaum, G. Cimò, L. Fuhrmann, \\ A. Kraus, A. Witzel and J. A. Zensus \\ Max-Planck-Institut für Radioastronomie, Auf dem Hügel 69, 53121 Bonn, Germany \\ tbeckert@mpifr-bonn.mpg.de \\ Received 2001 August 31, accepted 2001 November 20
}

\begin{abstract}
Radio variability on timescales from a few hours to several days in extragalactic flat spectrum radio sources is generally classified as intraday variability (IDV). The origin of this short term variability is still controversial and both extrinsic and intrinsic mechanisms must be considered and may both contribute to the observed variations. The measured linear and circular polarisation of IDV sources constrains the low energy end of the electron population. Any population of cold electrons within sources at or above the equipartition temperature of $10^{11} \mathrm{~K}$ depolarises the emission and can be ruled out. Intrinsic shock models are shown to either violate the large fraction of sources displaying IDV or they do not relax the light travel time argument for intrinsic variations. From structure function analysis, we further conclude that interstellar scintillation also leads to tight size estimates unless a very local cloud in the ISM is responsible for IDV.
\end{abstract}

Keywords: quasars: individual (0917+624) — ISM: structure — turbulence

\section{Introduction}

Intraday variability (IDV) of flat spectrum compact quasar cores and BL Lacs at $\mathrm{cm}$ wavelengths was discovered in 1985 (Heeschen et al. 1987) and is a common phenomenon ( $\sim 25 \%$ ) (Quirrenbach et al. 1992) among these sources. On rare occasions in $0716+714$ and $0954+658$, correlations with optical variations have been found and the evidence has been reviewed several times (e.g. Wagner \& Witzel 1995; Krichbaum et al. 2001). Radio-optical correlations, if real, suggest either fast intrinsic variations or gravitational lensing. From the light travel time argument the apparent brightness temperatures $T_{b}$ are in the range $10^{17}-10^{21} \mathrm{~K}$ and far in excess of the intrinsic inverse Compton (IC) limit of $10^{12} \mathrm{~K}$. The observed superluminal motions of jet components in many of these sources imply $\Gamma=5-10$ and allow for Doppler boosting factors $\mathcal{D}=(\Gamma(1-\beta \cos \theta))^{-1}$ and time shortening, which are insufficient for reducing the apparent $T_{b}$ down to the IC limit. The required Doppler factors $\mathcal{D}$ are in the range of 60 up to 1000 . Furthermore, jets with a surface brightness at the IC limit are radiatively inefficient (Begelman, Rees, \& Sikora 1994) and carry most of their energy as bulk motion. This is not supported by the observed power of radio lobes of these sources, and raises the energy requirement for the central engine to an uncomfortable level. It is therefore argued (Readhead 1994; Begelman 2001) that incoherent synchrotron sources in jets should radiate at the equipartition temperature $T_{E} \approx 10^{11} \mathrm{~K}$. This enforces Doppler factors which are 2-3 times larger than for the IC limit. Furthermore synchrotron sources at the IC limit with bulk motions of $\Gamma>100$ are dominated by IC scattering of either AGN photons (Begelman et al. 1994) or cosmic microwave background photons at redshifts $z \sim 1$ and not by the synchrotron self-compton process. The cooling is catastrophic, independent of the brightness temperature, and this explanation must therefore be discarded.

Other more tempting suggestions are the propagation of relativistic thin shocks in the jets (Qian et al. 1991), so that the observed variability timescale is not a measure of the source size, and scintillation induced by the interstellar medium (e.g. Rickett 1990) of otherwise nonvariable sources. We will explore both explanations in the following sections.

\section{Cool Particle Depolarisation}

The emission of incoherent synchrotron sources with high brightness temperatures is dominated by radiation from the $\tau=1$ surface. For intrinsic $T_{b}$ above the equipartition temperature, the energy of the source is dominated by particles $\left(e^{-}, e^{+}, p\right)$ with a strong dependence on $T_{b}$ :

$$
U_{e} / U_{B} \propto T_{b}^{7+2 \alpha},
$$

where $\alpha$ is the optically thin spectral index of the emission. Above the IC limit any contribution of cold electrons in an $e^{-} / p$ plasma will depolarise the synchrotron emission. Emission from the $\tau=1$ surfaces of flat spectrum radio cores comes predominantly from electrons with constant $\gamma_{\mathrm{rad}}$ factor, independent of frequency. We consider power law distributions of electrons $N(\gamma) \propto \gamma^{-p}$ above a lower cut-off $\gamma_{\text {min }}$. The degree of linear polarisation is likely to be reduced by tangled magnetic fields in a turbulent plasma with a typical wavenumber $k_{0}$ in sources of size $R$ (e.g. jet radius). For independent variations of magnetic field orientation in adjacent cells of size $k_{0}^{-1}$ the fractional linear polarisation is $\pi_{L} \propto\left(k_{0} R\right)^{-3 / 2}$. Further depolarisation inside one cell occurs, if Faraday rotation by electrons around $\gamma_{\min }$ (Jones \& O'Dell 1977) depolarises the radiation within the cell $\pi_{L} \propto \tau / \tau_{F}$. Here $\tau_{F}$ is the Faraday 
depth in the cell, which is smaller than the Faraday depth for the whole source by a factor $\left(k_{0} R\right)^{-1}$. The fractional linear polarisation can then be approximated by

$$
\pi_{L} \approx \frac{\alpha+1}{\alpha+5 / 3}\left(k_{0} R\right)^{-1 / 2}\left(\gamma_{\mathrm{rad}} / \gamma_{\min }\right)^{-p} \frac{\gamma_{\min }}{\ln \gamma_{\min }} .
$$

For sources above the IC limit the magnetic field strength must be very low and radiation at $\mathrm{GHz}$ frequencies is emitted by electrons with large $\gamma_{\mathrm{rad}}$. The resulting ratio $\gamma_{\mathrm{rad}} / \gamma_{\min }$ in equation (1) becomes large, and the polarisation drops below $1 \%$ if $\gamma_{\min }<10^{3}$ at $T_{b}=10^{12} \mathrm{~K}$. At equipartition temperature we still have depolarisation if $\gamma_{\min }<80$. The fact that most IDV sources are variable in polarised flux with a mean $\pi_{L} \geq 1 \%$ requires a cut-off in the electron population close to $\gamma$, which dominates the radiation from $\tau=1$ surfaces. At $T_{b}>10^{13} \mathrm{~K}$ this requires fine tuning of $\gamma_{\min }$ and provides strong constraints for acceleration mechanisms. Any substantial population of cold electrons with $\gamma_{\min } \approx 1$ is excluded in polarised sources with $T_{b}>10^{10} \mathrm{~K}$. This problem does not arise in pair plasma jets, because Faraday depolarisation does not occur there.

\section{The Alignment Problem of Thin Shock Propagation}

The thickness $\Delta z$ of a layer of post shock gas behind a relativistic shock, which travels with a shock Lorentz factor $\Gamma_{S}>2$ for a distance $z$ is

$$
\Delta z=\left(1-\sqrt{\frac{\Gamma_{S}^{2}-4}{\Gamma_{S}^{2}-1}}\right) z
$$

assuming that the gas leaves the shock at the sound speed and has an ultra-relativistic equation of state. A gas element that passed through the shock at $z=0$ is separated by a distance $\Delta z$ from the shock, when the shock has travelled a distance $z$ in the jet frame. For oblique shocks (as suggested by Spada, Salvati, \& Pacini 1999) the velocity and the thickness of the post shock gas will be larger. The ratio of thickness to the square root of the surface area is

$\tilde{\tau} \approx \Delta z /(\sqrt{\pi} z \sin \psi)=\left(1-\sqrt{\frac{\Gamma_{S}^{2}-4}{\Gamma_{S}^{2}-1}}\right) /(\sqrt{\pi} \sin \psi)$,

where it is assumed that the shock travelled at a constant velocity from the tip of a conical jet with half opening angle $\psi$. In the following we will assume that the shock travels only $1 / 10$ of that distance. When the shock is viewed face on, the surface area is a factor $1 / \tilde{\tau}^{2}$ larger than inferred from the variability timescale. But this factor is subject to relativistic aberration

$$
\tau=\mathcal{D} \sin \theta+\Gamma \mathcal{D} \tilde{\tau} \cos (\theta-\beta),
$$

where $\beta$ is the velocity of the post shock gas and $\theta$ the angle between jet and the line of sight. The true observed

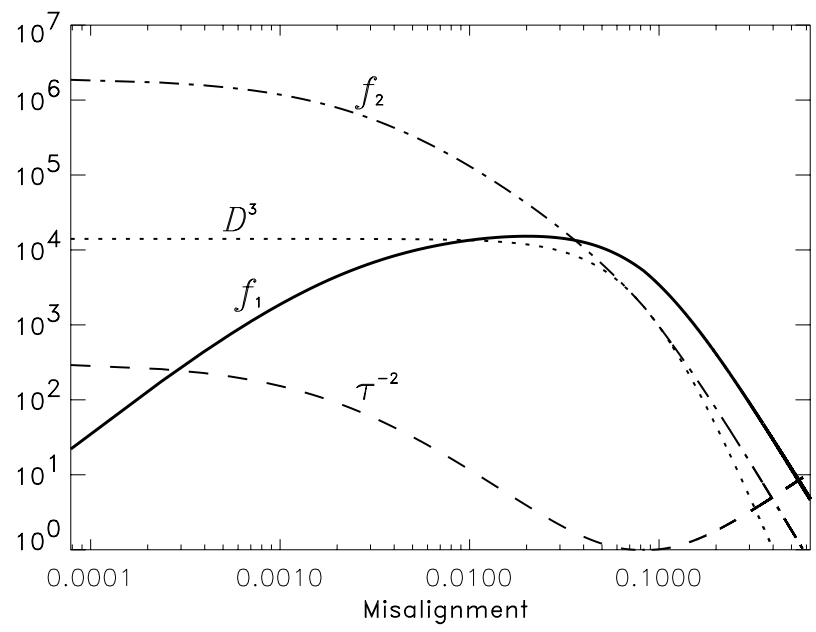

Figure 1 The square of the observable reduced timescale $\tau^{-2}$ (dashed) and the Doppler boost $\mathcal{D}^{3}$ (dotted) for the brightness temperature in the case of intrinsic variations are shown together with the flux-boosting factors $f_{1}$ (solid) and $f_{2}$ (dashed-dotted) as defined in the text. The misalignment is the angle $\theta$ between jet direction and line of sight. The assumed shock is travelling with $\Gamma_{S}=6$ in a jet of $\Gamma_{\text {pre }}=2.7$. The implied increase of observed $T_{b}$ due to orientation changes $f_{1}$ is not substantially larger than $\mathcal{D}^{3}$ and the result of scanned perturbations $f_{2}$ is only sufficient for explaining IDV for strong alignment of jet and observer.

flux variations can arise from orientation changes of shock and jet

$$
\frac{\Delta F_{\text {obs }}}{F_{\text {int }}}=f_{1}=3 \mathcal{D}^{2} \frac{\partial \mathcal{D}}{\partial \theta} \tau^{-2} \Delta \theta,
$$

or from inhomogeneities in particle density or magnetic field along the jet (Qian et al. 1991) which are scanned by the shock and travel at the speed of sound through the post shock gas

$$
\frac{\Delta F_{\text {obs }}}{F_{\text {int }}}=f_{2}=\mathcal{D}^{3}(\tau / 0.667)^{-2} .
$$

In Figure 1 the Doppler boosting of the flux due to intrinsic variations for a spherical source $\mathcal{D}^{3}$ is compared to the flux-boosting factors $f_{1}$ and $f_{2}$ for a shock with $\Gamma_{S}=6$ in a jet with $\psi=2.5^{\circ}$ and $\Gamma_{\text {pre }}=2.7$ for the preshocked gas (resulting in a post shock gas with $\Gamma=12$ ). Orientation changes of thin shocks require $\Delta \theta \sim \theta$ and consequently $f_{1}$ cannot substantially increase the flux variations on short timescales compared to variability in a spherical source. Only if disturbances along the jet are highlighted by the passage of a shock can the flux variations be so rapid and strong that $f_{2}$ exceeds $10^{6}$ which is necessary to bring a source with intrinsic $T_{b} \leq 10^{12} \mathrm{~K}$ up to the observed $10^{18} \mathrm{~K}$. Nonetheless this cannot provide an explanation for most IDV sources, because it requires a misalignment of the line of sight to the jet direction much smaller than $1 /(3 \Gamma)$, where the plateau of $\mathcal{D}^{3}$ starts (see Figure 1). In the specific case shown in Figure 1 the misalignment must be less than $10^{-3} \Gamma^{-1}$, which cannot be reconciled with $25 \%$ of core-dominated sources showing IDV. Furthermore, scanned perturbations in the jet lead to variable optical depth in the shocked gas and time lags, which are 


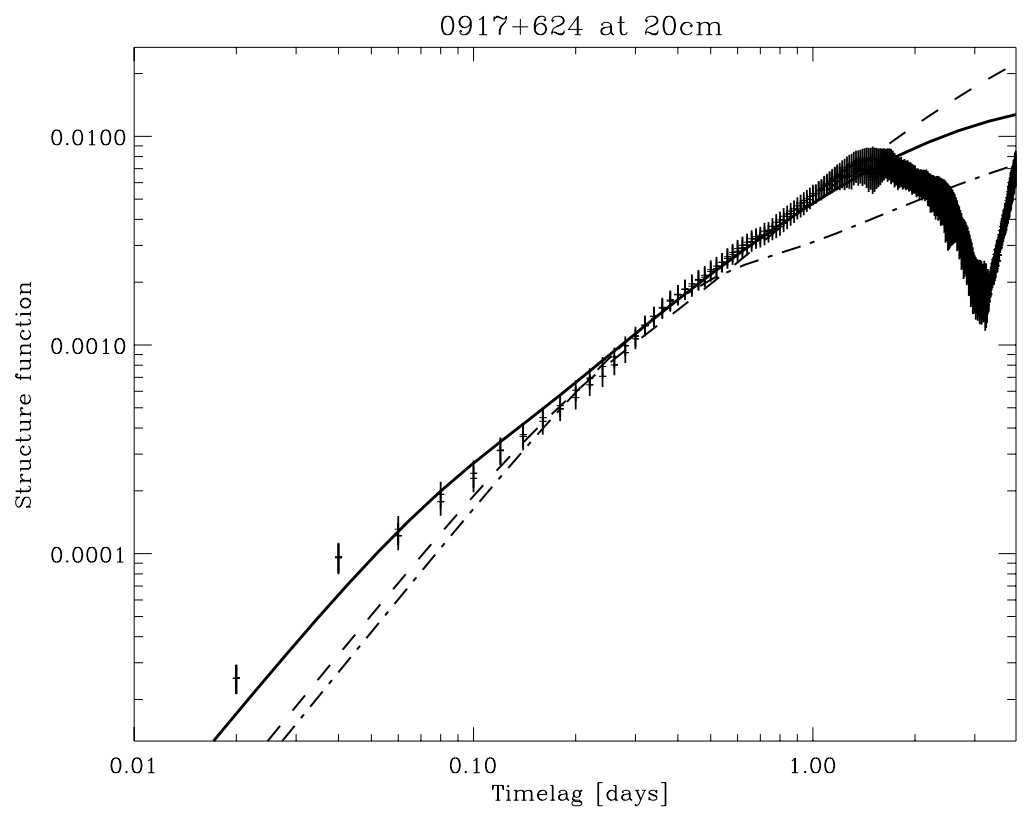

Figure 2 Structure function of total flux variations of $0917+624$ at $20 \mathrm{~cm}$ in 1989 . The data have recently been analysed by Qian et al. (2001). Over-plotted on the SF derived from the measurements are models for quenched scintillation according to Table 1 . The dashed-dotted line shows the model with a Kolmogorov spectrum $(\mathrm{K})$. The steep spectra models $(\mathrm{S} 1$ - dashed) and (S2 - solid) give better fits for the $20 \mathrm{~cm}$ data.

not observed in cross correlations of IDV at different radio frequencies.

\section{Refractive Interstellar Scintillation}

It is known from pulsar measurements that compact radio sources are subject to scintillation in the ISM of our Galaxy. Extragalactic sources flicker (Heeschen 1984) at frequencies $v \approx 1 \mathrm{GHz}$ with timescales of several days to weeks and this has been interpreted as a result of strong refractive scattering in the extended ISM of the Galactic disk (Blandford, Narayan, \& Romani 1986). The transition from strong to weak scattering is expected at about $5 \mathrm{GHz}$ depending on the path length in the disk towards the source. The maximum of the modulation index $m$ is $\approx 1$ for a point source. A 1 Jy source with an apparent $T_{b}$ of $10^{12} \mathrm{~K}$ has a size of $230 \mu$ as at $\lambda 6 \mathrm{~cm}$, and scintillation will be quenched ${ }^{1}$ because the source size is much larger than the angular size of the Fresnel scale in the ISM. For a typical distance of $200 \mathrm{pc}$ to the scattering medium, the expected modulation index is $m \leq 1.7 \%$, while a source with $T_{b}=10^{13} \mathrm{~K}$ will have $m \leq 5 \%$ with a characteristic timescale of 0.5 days. Therefore any $1 \mathrm{Jy}$ source with apparent $T_{b}$ above a few $\times 10^{12} \mathrm{~K}$ is expected to scintillate with a timescale comparable to those observed in IDV sources, as has been demonstrated by Rickett et al. (1995) for $0917+624$.

The light curves of scintillating sources contain further information, which can be explored via the structure function (SF) (Simonetti, Cordes, \& Heeschen 1985):

$$
\mathrm{SF}(\tau)=\left\langle[I(t+\tau)-I(t)]^{2}\right\rangle_{t} .
$$

\footnotetext{
${ }^{1} \mathrm{~A}$ Gaussian brightness distribution is assumed for extended sources in this paper.
}

The SF extracted from the data can be compared to theoretical expectations. For strong refraction and weak scintillation in an extended medium, Coles et al. (1987) gave a closed form for SFs, which includes three cut-offs for the Fourier transform of the local SF. These are due to the Fresnel scale, the visibility amplitude, which contains the size of the source, and an exponential cut-off in strong scintillation giving rise to the refractive scale. Whichever comes first determines the modulation index and the slope of the SF below the first maximum (see Figure 2). Following Blandford et al. (1986) we assume a Gaussian distribution of ionised matter, keeping in mind that for IDV a much smaller scale height $H \sim 100 \mathrm{pc}$ than the ISM scale height in the Galactic disk is required.

We concentrate on the modulation index derived from the plateau of the SF at large time lags and on the slope for shorter lags. It turns out that in weak scintillation the slope of the SF is either quadratic, $\mathrm{SF}(t) \propto t^{\alpha}, \alpha=2$ for steep turbulent spectra $\beta \geq 4$, or $\alpha=\beta-2$ for $\beta<4$.

In strong and quenched scintillation the SF shows a broken power law below the maximum and this slope also depends on the power law index $\beta$ of the turbulent spectrum $\Phi(q)=C_{N}^{2} q^{-\beta}$ of density fluctuations in the ISM. For the limiting case $\beta=4$ discussed in Blandford et al. (1986), this slope is $\alpha=1$ in the quenched and strong case, while for a Kolmogorov-like spectrum $\beta=11 / 3$ the slope is $\alpha=5 / 6$ for strong scattering and $\alpha=2 / 3$ for quenched scintillation. For quenched scintillation, the slope is generally $\alpha=\beta-3$.

We have computed theoretical structure functions dominated by quenched scintillation and compared them to the observed data taken at $\lambda 20 \mathrm{~cm}$ and $\lambda 6 \mathrm{~cm}$ (Figures 2 and 3). The parameters are the power law index $\beta$, the strength of density fluctuations $C_{N}^{2}$, the scale height $H$, 


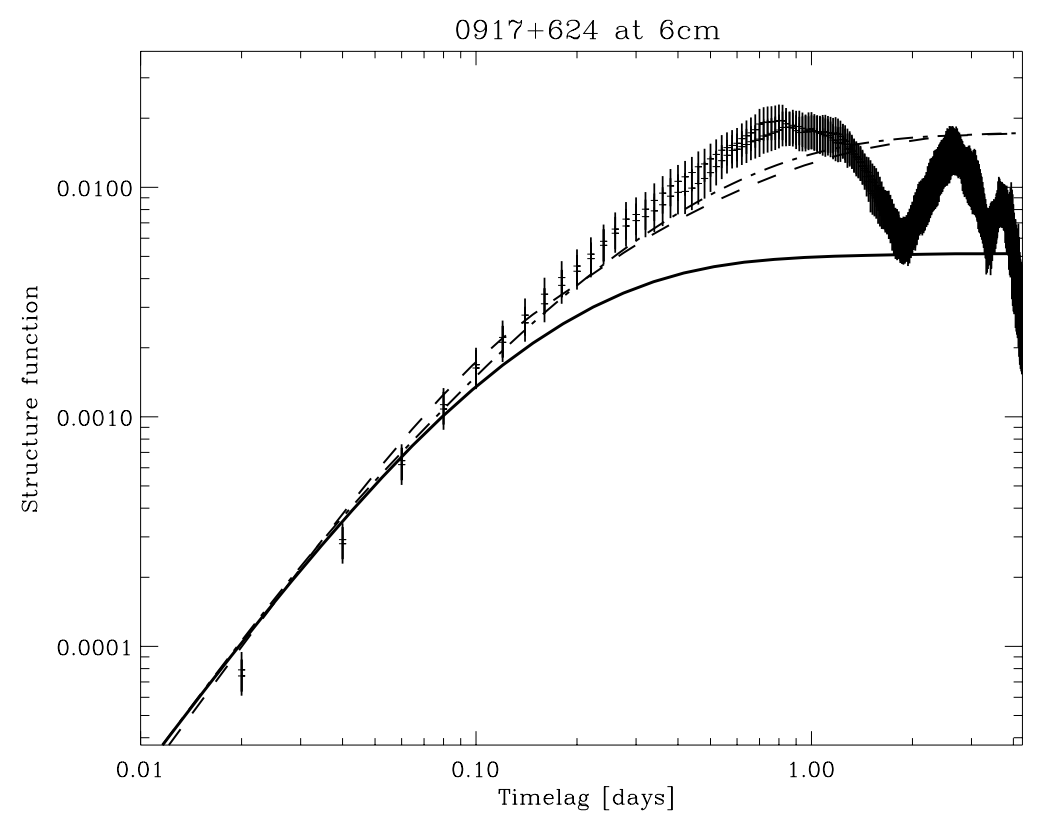

Figure 3 Structure function of total flux variations of $0917+624$ at $6 \mathrm{~cm}$ in 1997. The data come from one of the best sampled light curves at this wavelength. Over-plotted on the SF derived from the measurement are the same models for quenched scintillation as in Figure 2 applied to $\lambda 6 \mathrm{~cm}$.

Table 1. Parameters for the structure function models. The result for $\lambda 6 \mathrm{~cm}$ and $\lambda 20 \mathrm{~cm}$ are shown in Figures 2 and 3. The angular size $\theta_{\mathrm{S} 6}$ and $\theta_{\mathrm{S20}}$ are the source size at $\lambda 6 \mathrm{~cm}$ and $\lambda 20 \mathrm{~cm}$

\begin{tabular}{lcccccc}
\hline Model & $H(\mathrm{pc})$ & $\beta$ & $C_{N}^{2}\left(\mathrm{~m}^{-\beta-3}\right)$ & $\theta_{S 6}(\mu \mathrm{as})$ & $\theta_{S 20}(\mathrm{mas})$ & $v(\mathrm{~km} / \mathrm{s})$ \\
\hline K & 70 & $11 / 3$ & $10^{-3}$ & 40 & 0.4 & 5 \\
S1 & 70 & 4.567 & $10^{-10.6}$ & 40 & 0.16 & 14 \\
S2 & 140 & 4.567 & $10^{-12.15}$ & 40 & 0.16 & 30 \\
\hline
\end{tabular}

and the velocity of the observer relative to the ISM cloud. From simultaneous fitting of the SFs at $6 \mathrm{~cm}$ and $20 \mathrm{~cm}$ we can find plausible parameters and the size of the source at these wavelengths. The model parameters are summarised in Table 1. It is clearly visible from Figures 2 and 3 that a steep spectrum is preferred.

In particular the slope $\alpha \approx 1.3$ at $\lambda 20 \mathrm{~cm}$ indicates a steep spectrum $\beta=4.3$, when only quenched scintillation is assumed. The turbulent spectrum has to be even steeper because the SF flattens before turning over into the $\alpha=$ $\beta-2$ slope dictated by the Fresnel cut-off at smaller time lags. Together with the weak curvature near the plateau for large time lags, this points to the slope $\beta \approx 4.6$ of the models S1 and S2.

\section{Conclusions}

Based on IDV observations of several quasar and BL Lac radio cores with apparent brightness temperatures in the range $10^{16}-10^{21} \mathrm{~K}$ we investigated the possibility of intrinsic variations due to the propagation of thin relativistic shocks. We find that no source with $T_{b}>10^{16} \mathrm{~K}$ can be explained by that model without calling for Doppler factors larger than 20 and strong alignment between the jet and the line of sight. The required alignment firmly rules out this hypothesis as an explanation for all IDV sources.

Based on refractive interstellar scintillation IDV can be explained by turbulence in the ISM. The scale size of the ionised gas responsible for IDV is about $100 \mathrm{pc}$ in the case of $0917+624$, and scintillation is quenched by the source size, which is larger than the refractive or Fresnel scale in the ISM. A fit to the structure functions at $\lambda 20 \mathrm{~cm}$ and $\lambda 6 \mathrm{~cm}$ indicates a steep power law $(\beta \approx 4.6)$ for $\Phi(q)$ fluctuations. This corresponds to an energy spectrum $\sim q^{-2.6}$ that is much steeper than both a Kolmogorov spectrum and the $q^{-2}$ spectrum for compressible turbulence, but a bit shallower than $\sim q^{-3}$ expected for twodimensional turbulence. The length scale probed in the ISM by these measurements are between $5 \times 10^{8} \mathrm{~m}$ and $2 \times 10^{9} \mathrm{~m}$ at the peak of the structure functions. The slope of the turbulent spectrum is derived from structure functions at small time lags and the corresponding spectrum extends an order of magnitude down to smaller spatial scales. Compressible turbulence is not unexpected at these scales, if turbulence is driven by shocks from supernovae or by stellar winds.

Scintillation in $0917+624$ is quenched by the source size, which is one parameter of the theoretical fits to the 
SFs. The required sizes are $\sim 40 \mu$ as and $\sim 0.4$ mas at $\lambda 6 \mathrm{~cm}$ and $\lambda 20 \mathrm{~cm}$ respectively. At $\lambda 6 \mathrm{~cm}$, the flux of $0917+624$ (redshift $z=1.446$ ) is $1.5 \mathrm{Jy}$. Combined with the derived source size this implies $T_{b}=10^{14} \mathrm{~K}$. Again Doppler factors of about 100 are needed to avoid the IC catastrophe or Doppler factors of 1000 to arrive at equipartition temperature. The sizes derived for $0917+624$ from structure function models might be changed, if a degeneracy in the model parameters exists. The most plausible direction is an even closer ISM screen with a higher level of turbulence. In this case the angular Fresnel scale gets larger and larger source sizes are allowed.

\section{References}

Begelman, M. C. 2001, PASA, 19,

Begelman, M. C., Rees, M. J., \& Sikora, M. 1994, ApJ, 429, L57

Blandford, R., Narayan, R., \& Romani, R. W. 1986, ApJ, 301, L53
Coles, W. A., Frehlich, R. G., Rickett, B. J., \& Codona, J. L. 1987, ApJ, 315, 666

Heeschen, D. S. 1984, AJ, 89, 1111

Heeschen, D. S., Krichbaum, T. P., Schalinski, C. J., \& Witzel, A. 1987, AJ, 94, 1493

Krichbaum, T. P., Kraus, A., Fuhrmann, L., Cimò, G., \& Witzel, A. 2001, PASA, 19, 14

Jones, T. W., \& O’Dell, S. L. 1977, ApJ, 214, 522

Qian, S. J., Quirrenbach, A., Witzel, A., Krichbaum, T. P., Hummel, C. A., Zensus, J. A. 1991, A\&A, 241, 15

Qian, S. J., Witzel, A., Kraus, A., Krichbaum, T. P., Zensus, J. A. 2001, A\&A, 367, 770

Quirrenbach, A., et al. 1992, A\&A, 258, 279

Readhead, A. C. S. 1994, ApJ, 426, 51

Rickett, B. J. 1990, ARA\&A, 28, 561

Rickett, B. J., Quirrenbach, A., Wegner, R., Krichbaum, T. P., \& Witzel, A. 1995, A\&A, 293, 479

Simonetti, J. H., Cordes, J. M., \& Heeschen, D. S. 1985, ApJ, 296, 46

Spada, M., Salvati, M., \& Pacini, F. 1999, ApJ, 511, 136

Wagner, S. J., \& Witzel, A. 1995, ARA\&A, 33, 163 